\title{
Discrete element simulation: challenges in application and model calibration
}

\author{
Avi Levy · Jin Y. Ooi
}

Received: 14 December 2010 / Published online: 1 February 2011

(C) Springer-Verlag 2011

Bulk handling and processing of particulate materials are integral to a wide range of industrial processes. Many of these industrial particulate systems display difficult and unpredictable behaviour, giving rise to considerable challenges in the design and operation of the plants. In recent decades, the discrete element method (DEM), which models the motion and interaction of individual particles, has become increasingly popular as a computational tool to study and investigate complex particulate systems. To date, considerable scientific advances have been made in the development of the technique, resulting in an explosion of the use of DEM in a myriad of industrial and scientific applications. However, careful validation of simulation results with closely matching experimental data are still rather rare and it is vital to establish DEM's capability to produce satisfactory quantitative predictions.

The popularity of DEM can be discerned from the rapidly increasing number of DEM related papers being offered at the leading international conference on the bulk handling of particulate solids. This Special Issue comprises a series of papers that have been selected from the 6th International Conference for Conveying and Handling of Particulate Solids (CHoPS 2009) which was held in Brisbane, Australia, in August 2009. The papers within these

\footnotetext{
A. Levy $(\varangle)$

Pearlstone Centre for Aeronautical Engineering Studies,

Department of Mechanical Engineering, Ben-Gurion University

of the Negev, P.O. Box 653, Beer-Sheva 84105, Israel

e-mail: avi@bgu.ac.il

J. Y. Ooi

Institute for Infrastructure and Environment, School of Engineering, University of Edinburgh, Edinburgh, EH9 3JL, UK

e-mail: j.ooi@ed.ac.uk
}

covers represent a significant effort by the authors in deploying DEM to study a range of industrial problems, to investigate the calibration of DEM models and to provide further insight into the mechanics governing these granular processes. These papers demonstrate clearly the considerable potential of this powerful numerical technique to provide innovative solutions. They also highlight the challenges that must be overcome to transform DEM from a largely scientific tool into an effective industrial predictive tool, which will require a concerted effort from both the scientific and engineering communities. It is therefore most appropriate that this special issue should appear in Granular Matter which has its readership reaching both communities. Due to a production error, one of the papers invited for this Special Issue ("Application of DEM to evaluate and compare process parameters for a particle failure under different loading conditions" submitted by Manoj Khanal and Jürgen Tomas) was already published as Ref. [1].

Finally, we would like to express our sincere thanks to all the authors for their contributions and to the Granular Matter Editors-in-Chief for making this special issue possible. We would also like to this opportunity to encourage all researchers and industrial practitioners who are interested in DEM research and application to contribute to the 7th International Conference for Conveying and Handling of Particulate Solids-CHoPS 2012 which will be held in September 2012 at Friedrichshafen, Germany.

\section{Reference}

1. Khanal, M., Tomas, J.: Application of DEM to evaluate and compare process parameters for a particle failure under different loading conditions. Granular Matter 12(4), 411-416 (2010) 